\title{
Relationship between positive clinical VTO and post-treatment soft tissue profile following phase I growth modification therapy
}

\author{
Dr Shabbir Hussain Shah,' Dr Muhammad Azeem,2 Dr Waheed Ul Hamid, ${ }^{3}$ Dr Faiz Rasool4 \\ 'Assistant Professor, ${ }^{3}$ Principal and Professor, Dept of Orthodontics, ${ }^{A}$ Assistant Professor, Dept Community \& Preventive Dentistry, \\ de'Montmorency College of Dentistry, Pakistan \\ ${ }^{2}$ Assistant Professor, Dept. of Orthodontics, Dental Section-Faisalabad Medical University/Punjab Medical College, Pakistan.
}

\section{ABSTRACT}

Introduction: Facial profile improvement is goal of cotemporary orthodontics and a reason to seek orthodontic therapy. The soft tissue profile plays a important role on orthodontic diagnosis and treatment planning. The objective of this study is to investigate the relationship between positive clinical VTO and actual post-treatment soft tissue profile after phase I therapy of growth modification in Class II.

Materials \& Method: Pretreatment simulation of post-treatment and actual post-treatment profile photographs of 30 class $\|$ div I patients treated with twin block appliance were compared. Three profile photographs of each subject; pretreatment, positive clinical VTO and post-treatment were taken and on each photograph four angles; Nasofacial (NF), Nasomental (NM), Mentocervical (MC) and Nasolabial (NL) were drawn and measured. Mean, standard deviation, success and coefficient of determination of each angle was measured and linear regressions analysis was applied to find out the correlation.

Result: Nasolabial and nasomental angles showed greater success i.e. $81.4 \%$ and $68.1 \%$ respectively showing greater correlation, while nasofacial and mentocervical angles showed less success i.e. $48.1 \%$ and $48.3 \%$ respectively showing less correlation. Linear regression analysis revealed that positive clinical VTO significantly predicted post-treatment profile whereas coefficient of determination for nasomental and mentocervical angles was $76.5 \%$ and $60 \%$ representing a better goodness of fit while nasolabial and nasofacial angles was $53.6 \%$ and $51.6 \%$ demonstrating poor fit of regression lines.

Conclusion: Even though there is improved facial profile obtained by protracting the mandible into class I relation in a chair side maneuver in class II div I malocclusions, yet the orthodontist should be tentative when predicting the outcome of growth modification to get benefit of this therapy.

Key-words: Class II; Growth modification; Predication; VTO.

\section{INTRODUCTION}

Growing subjects with angle's class II malocclusion with deficient mandible exhibit convex profile as compared to normal subjects.' In keeping with Mcnamara, class II malocclusion in preadolescent subjects is mostly comprised of mandibular retrognathia, ${ }^{2}$ whereas dentofacial distortion can have considerable impact on psychological wellbeing of an individual. ${ }^{3}$

Facial profile improvement is goal of contemporary orthodontics and a reason to seek orthodontic therapy. Treatment that alters facial profile by addressing the skeletal imbalance is of great interest ever since. ${ }^{4}$ In growing subjects with class II malocclusion, two phase therapy including growth modification followed by comprehensive orthodontic treatment is advocated. For this purpose functional appliances are used to correct the mandibular deficiency in phase I therapy of growth modification. Appliances are used for the period of 9 to 12 months around the pubertal growth spurt in addition to appropriate retentive period. ${ }^{5}$
During orthodontic treatment planning, facial growth prediction is of great significance. This can be performed conveniently with the help of templates, ${ }^{6}$ and computer software algorithms for instance Bolton and Ricketts. ${ }^{7}$ In class || div | malocclusion, mandibular protraction into class I relation improves the facial profile and eases the air way. ${ }^{8}$ This chair side method to demonstrate profile improvement by protracting the mandible into class I relation is recognized as positive clinical VTO and the patient is anticipated to get benefit of growth modification therapy. ${ }^{9}$

The aim of present study was to find out the relationship of the post-treatment soft tissue profile simulation based on intentional mandibular protraction into visually appealing facial shape from retruded position and actual posttreatment outcome. To our knowledge no such study exists so far in literature on this topic. This prediction planning of growth modification procedure can enable orthodontist and patient to visualize the expected post-treatment effect of a treatment modality. 


\section{MATERIALS AND METHOD}

The study protocol was approved by ethical committee. Sample was retrieved from the past work done in Orthodontic department concerning growth modification in class II div I malocclusions.

The selection criteria for this investigation was (1) photographs of subjects with class II div I malocclusion chosen to be treated with twin block appliance, (2) Initial, positive clinical VTO and post-treatment profile photographs following the completion of phase-l therapy, (3) No relevant medical or dental history, (4) No previous history of orthodontic treatment.

Methodology was designed to take black and white profile photographs of the 30 class II div I patients treated with twin block appliance on A4 size white papers. Pretreatment, simulation of posttreatment profile and actual posttreatment profile photographs of each patient were considered. This view provides better idea concerning facial changes in saggital direction followed by FA therapy.

All photograph selected were with Frankfort horizontal plane parallel to the ground. Pretreatment photographs were selected with deficient mandibular position and relaxed lips, simulation photographs with protracted mandibular position with closed lips to achieve harmonious profile and post-treatment photograph of favorable FA therapy result with relaxed lips.

Photographs were edited in such a way that head of each patient had the same size. Only angular measurements of profile view were taken which were supposed to be less affected by size of the photograph contrary to liner measurements.

For each subject three profile photographs; pretreatment, positive clinical VTO and posttreatment were taken.
Four facial contour angles; Nasofacial, Nasomental, Mentocervical and Nasolabial were drawn and measured on each photograph. These angles are notably influenced by change in profile. Mean, standard deviation, success and coefficient of determination of every angle was measured to identify existence of whichever relationship linking them.

Treatment group comprised of 10 female and 20 male patients. All were at the peak pubertal growing stage. Removable standard twin block appliances were used for growth modification purpose. Average treatment time was 18 months inclusive of retentive period.

\section{RESULT}

Post-treatment profile was significantly less improved than the actual post-treatment simulation profile.

Mean, standard deviation, success and coefficient of determination for all angular measurements are summarized in tables 1, 2, 3 and 4. In general, two variables NF angle and MC angle showed less success $48.1 \%$ and $48.3 \%$ respectively less predictive of final outcome, while other two variables NM angle and NL angle showed greater success $68.1 \%$ and $81.4 \%$ respectively more predictive of final outcome.

Mean pre-treatment nasofacial angle was $36.53^{\circ} \pm 3.889$ and simulation was $31.27^{\circ} \pm 4.026$ while mean posttreatment was $34^{\circ} \pm 4.472$ and the success was $48.1 \%$. Mean pre-treatment nasomental angle was $122.07^{\circ} \pm$ 4.743 and simulation was $128.13^{\circ} \pm 5.125$ while mean post-treatment was $126.20^{\circ} \pm 4.648$ and the success was $68.1 \%$. Mean pre-treatment mentocervical angle was $99.07^{\circ} \pm 8.71$ and simulation was $87.47^{\circ} \pm 10.18$ while mean post-treatment was $93.47^{\circ} \pm 7.05$ and the success was $48.3 \%$. Mean pre-treatment nasolabial angle was $94.40^{\circ}$ \pm 8.94 and simulation was $101.20^{\circ} \pm 9.63$ while mean posttreatment was $99.93^{\circ} \pm 11.33$ and the success was $81.4 \%$.

Table 1: Regression analysis: Predictors $=$ Nasofacial Simulation; Dependent Variable $=$ Nasofacial Post-treatment

\begin{tabular}{|l|c|c|c|c|c|c|}
\hline \multicolumn{1}{|c|}{ Sample } & Mean & SD & $\begin{array}{c}\text { Expected Change } \\
\text { (Pre-treatVTO) }\end{array}$ & $\begin{array}{c}\text { Achieved } \\
\text { (Pre-Post) }\end{array}$ & Success & R square* \\
\hline Nasofacial Pretreatment & 36.53 & 3.889 & A & B & B/A*100 & $51.6 \%$ \\
\hline Nasofacial Simulation & 31.27 & 4.026 & 5.26 & & $48.1 \%$ \\
\hline Nasofacial Posttreatment & 34.00 & 4.472 & & 2.53 & \\
\hline
\end{tabular}

Table 2: Regression analysis: Predictors $=$ Nasomental Simulation; Dependent Variable $=$ Nasomental Post-treatment

\begin{tabular}{|l|c|c|c|c|c|c|}
\hline \multicolumn{1}{|c|}{ Sample } & Mean & SD & $\begin{array}{c}\text { Expected Change } \\
\text { (Pre-treatVTO) }\end{array}$ & $\begin{array}{c}\text { Achieved } \\
\text { (Pre-Post) }\end{array}$ & Success & R square* \\
\hline Nasomental Pretreatment & 122.07 & 4.743 & A & B & B/A*100 & $76.5 \%$ \\
\hline NasomentalSimulation & 128.13 & 5.125 & -6.07 & & $68.1 \%$ \\
\hline NasomentalPosttreatment & 126.20 & 4.648 & & -4.13 & \\
\hline
\end{tabular}


Table 3: Regression analysis: Predictors $=$ Mentocervical Simulation; Dependent Variable $=$ Mentocervical Post-treatment

\begin{tabular}{|l|c|c|c|c|c|c|}
\hline \multicolumn{1}{|c|}{ Sample } & Mean & SD & $\begin{array}{c}\text { Expected Change } \\
\text { (Pre-treatVTO) }\end{array}$ & $\begin{array}{c}\text { Achieved } \\
\text { (Pre-Post) }\end{array}$ & Success & R square* \\
\hline Mentocervical Pretreatment & 99.07 & 8.71 & A & B & B/A*100 \\
\hline MentocervicalSimulation & 87.47 & 10.18 & 11.60 & & $48.3 \%$ \\
\hline MentocervicalPosttreatment & 93.47 & 7.05 & & 5.60 & $6.0 \%$ \\
\hline
\end{tabular}

Table 4: Regression analysis: Predictors $=$ Nasolabial Simulation; Dependent Variable $=$ Nasolabial Post-treatment

\begin{tabular}{|l|c|c|c|c|c|c|}
\hline \multicolumn{1}{|c|}{ Sample } & Mean & SD & $\begin{array}{c}\text { Expected Change } \\
\text { (Pre-treatVTO) }\end{array}$ & $\begin{array}{c}\text { Achieved } \\
\text { (Pre-Post) }\end{array}$ & Success & R square* \\
\hline Nasolabial Pretreatment & 94.40 & 8.94 & A & B & B/A*100 \\
\hline NasolabialSimulation & 101.20 & 9.63 & -6.80 & & $81.4 \%$ \\
\hline NasolabialPosttreatment & 99.93 & 11.33 & & -5.53 & $53.6 \%$ \\
\hline
\end{tabular}

\section{DISCUSSION}

Class II malocclusion constitutes a significant part of all malocclusions in a society which are presented to practicing orthodontists. Large portion of class II patients have a significant skeletal imbalance and much of orthodontic treatment is aimed at correcting or masking this discrepancy. ${ }^{10}$ In growing patient success of the treatment lies on the ability of clinician to influence growth changes in maxilla and mandible. Child with jaw discrepancy can have impaired esthetics which must be eradicated to deal with social stigma. Another indication for treatment in such subjects is the dental and skeletal profile which is susceptible to trauma owing to increased overjet and protrusive incisors. ${ }^{11}$

Excellent data is available from clinical trials in the literature regarding growth modification. But it remained difficult to predict individual response to a particular treatment plan since growth is unremarkably unpredictable. ${ }^{6}$ In one study to investigate perceived facial changes of class II div I subjects with convex profile after functional orthopedic treatment, it was revealed that facial profiles were improved but quite limited. Thus the care giver should be tentative when predicting the outcome of functional therapy. ${ }^{12}$ In another study profile silhovettes were sued to investigate change in profile attractiveness after FA therapy. It was concluded that FA do not lead to more attractive profile than control group. ${ }^{13}$ But in one study utilizing twin block functional appliance treatment to observe improvement in profile attractiveness, silhovettes were used. Facial profile was perceived to be more attractive in treatment group by raters than those who didn't received treatment. ${ }^{14}$
In various studies clinical VTO was performed to show the positive anticipated treatment results with functional appliance therapy in growing class || div I subjects. ${ }^{15,16}$ But no such relationship was observed linking the anticipated treatment result and the actual treatment outcome in profile harmony. The aim of present study was to find out the relationship of the post-treatment soft tissue profile simulation based on intentional mandibular protraction into visually appealing facial shape from retruded position and actual post-treatment outcome.

In this study, profile photographs were used to focus closely where functional treatment had its maximum effect. Photographs were edited in such a manner so that the head of each patient had the same size in a set of 3 photographs i.e. before treatment, with positive clinical VTO and after treatment.

We used profile photograph of clinical chair side maneuver to watch the anticipated profile improvement by protracting the mandible in previously treated FA candidates of class II div I malocclusions. Only the successfully treated patients were taken. Selected patients got their profile improved by forward repositioning of mandible and ultimately took reasonable benefit from the growth modification therapy. However there is variation between profile simulation and actual profile changes in terms of facial angles.

More of the similarities were along nasomental and nasolabial angles. These being able to visualize the treatment outcome with less inaccuracy. While fewer similarities were along nasofacial and mentocervical angles. These illustrating weak association linking the prediction and the end result. 
Sample size was considered adequate to discover such a relationship in accordance with other profile studies, foreshowing improvement in profile following FA therapy. To our knowledge, no author has attempted to investigate the relationship between post-treatment soft tissue profile simulation and actual post-treatment soft tissue profile following phase I therapy of growth modification in class || div | malocclusion. While projected changes in jaw position in a computer generated simulation of post treatment profile are source of visualization of treatment effect by doctor and patients. 6

In medicine significant progress has been made about the visualization methods to support computerexecutable treatment plans to investigate the effects of the therapy on patient condition. ${ }^{17}$ Rickett's long term growth prediction method based on baseline cephalometrics values conducted on Turkish children revealed that it was helpful in treatment planning after comparison with actual growth 7 years later. ${ }^{18}$ In another study various methods of prediction were discussed. A longitudinal method which is based on annual x-ray cephalograms is shown to be of limited use, as the remodeling process at the lower border of the mandible to a large extent masks the actual rotation. A metric method based on a metric description of the facial morphology at a single stage of development, has so far not proved of value. A structural method is described by which prediction is possible from a single cephalograms. This method is based on information gained from implant studies of the remodeling process of the mandible during growth. ${ }^{19}$ Mori developed a simulation system for mandibular orthognathic surgery. This technique is based on integrated three dimensional data and showed acceptable precision of treatment planning for orthognathic surgery, especially for facial asymmetry. ${ }^{20}$

Kosh presented prediction of post surgical facial shape which is paramount importance in facial esthetic surgical planning. This procedure is based on volumetric finite element modeling. Constructions enable us to compute smooth and visually appealing facial shapes with numerical accuracies. ${ }^{21}$ However, visualizing the effects of applying treatment plans as well as supporting the exploration of effects on the patient's condition are still largely unresolved tasks. ${ }^{17}$

\section{CONCLUSION}

Nasolabial angle and nasomental angle show positive correlation with post treatment change however nasofacial and mentocervical angles have poor prediction. Even though there is improved facial profile obtained by protracting the mandible into class I relation in a chair side maneuver in class || div | malocclusions, yet the orthodontist should be cautious when predicting the outcome of growth modification to get benefit of this therapy.

\section{REFERENCES}

1. Singh GD, Clark WJ. Soft tissues changes in patients with class II div I malocclusions treated using Twin Block appliance: finite element scaling analysis. Eur J Orthod 2003;25:225-230.

2. McNamara Jr JA. Components of class II malocclusion in children 8-10 years of age. Angle orthod 1981 jul;51 (3):177-202

3. Motloba DP, Sethusa MPS, Ayo-Yusuf OA. The psychological impact of malocclusion on patients seeking orthodontic treatment at a south African Oral Health Training Centre. SADJ 2016 june;71 (5):200-205

4. Tulloch JF, Medland W, Tuncay OC. Methods used to evaluate growth modification in class II malocclusion. Am J Orthod Dentofacial Orthop 1990:98:340-7

5. Nayak KU, Goyal V, Malviya N. Two-phase treatment of class II malocclusion in young patient. Contemp Clin Dent 201 1;2:376-380

6. Proffit WR, Fields HW, Sarver DM. Contemporary Orthodontics. 5th ed. Mosby: Elsevier; 2012. 239p

7. Sangun M, Kusnoto B, Evans CA, Galang-Bouiren MT, Vaina G, Obrez A. Evaluation of Ricketts' and Bolton's growth prediction algorithms embedded in two diagnostic imaging and cephalometric software. ournal of the World Federation of Orthodontists 2015;4:146-150

8. Graber LW, Vanarsdall RL, Vig KW, Huang GJ. Orthodontics: current principals and techniques. 6th ed. St. Louis, Missouri: Elsevier; 2017. 997p

9. Rai AK.Determenent of a successful case: Clinical changes or cephalometric readings? Class II division I correction using Forsus fatigue resistant device. Dentistry 2014;4:10

10. Naragond A, Kenganal S, Sagarkar R, Sugaradday. Orthodontic camouflage treatment in an adult patient with a class II, division I malocclusion- A case report. J Clin Diagn Res 2013;7:395-400 
11. Proffit WR, Fields HW, Sarver DM. Contemporary Orthodontics. 5th ed. Mosby: Elsevier; 2012. 475p

12. Tsiouli K, Topouzelis N, Papadopoulos MA, Gkantidis N. Perceived facial changes of class II division I patients with convex profiles after functional orthopedic treatment followed by fixed orthodontic appliances. AJO-DO 2017;152:80-91

13. O'Neil K, Harkness M, Knight R, Rating of the profile attractiveness after functional appliance treatment. AJO-DO 2000; 1 18:371-6: discussion, 377.

14. O'Brien K, Macfarlane T, Wright J, Conboy F, Appelbe P, Birnie D, et al. Early treatment for class II malocclusion and perceived improvement in facial profile.AJO-DO 2009;135:580-5

15. Mahadevia SM, Assudani NP, Gowda K, Joshipura AJ. Twin star: Adding a new dimention for treatment of class II noncompliant patients. APOS Trends in orthodontics 2018;4:21-5

16. Sharma NS. Management of growing skeletal class II patient: A case report. International jounal of clinical pediatric dentistry 2013;6:48-54

17. Gschwandtner T. Interactive Visualization of Effects of Medical Treatment on a patient's Condition.\{dissertation\}. Vienna University of Technology;2012.140 p.

18. Kocadereli I, Telli AT. Evaluation of Ricketts' long-range growth prediction in Turkish children. AJO-DO 1999;1 15:515-520

19. Björk A. Prediction of mandibular growth rotation. AJO-DO 1969;55:585-599

20. Mori Y, Shimizu H, Minami K, Kwon T, Mano T. Development of a simulation system in mandibular orthognathic surgery based on integrated three-dimensional data. Oral Maxillofac Surg 2011; 15:131-138

21. Koch RM, Roth SM, Gross MH, Zimmermann AP, Sailer HF. A framework for facial surgery simulation. InProceedings of the 18th spring conference on Computer graphics 2002 Apr 24 (pp. 33-42). ACM. 